\title{
Simulated Scalar Feed Horn Antenna with Exterior Tapered Throat Profile
}

\author{
Dr. Stephen Rodrigues \\ Department of Instrumentation, Cochin University of Science \& Technology, Cochin-682 022, India.
}

\begin{abstract}
Simulated scalar feed horns are capable of giving radiation characteristics of identical metallic corrugated feed horns. The impedance matching tapered throat profile of these horns is provided to the interior side of the E-plane walls. In the present study, by avoiding the abrupt angular variation caused by the interior tapered profile structure at the throat region, the possibility of obtaining better impedance matching is explored by giving the tapered throat profile to the exterior side of the E-plane walls. The radiation characteristics of the new horn are analyzed by comparing its radiation characteristics with that of an identical horn with interior throat profile structure. Though considerable improvement is not achieved in return-loss characteristics, improvements in side lobe, back lobe levels and gain are achieved.
\end{abstract}

Key Words - Microwaves, Feed horns, corrugated feed horns, dielectric loaded feeds, and simulated feed orns

\section{Introduction}

Metallic corrugated horn antennas, by virtue of its radiation characteristics, [6] are found to be excellent feeds for large reflector antennas. The main disadvantages of these antennas are their high production

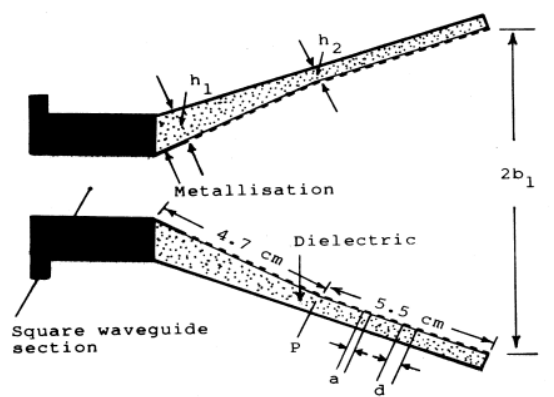

Horn HI

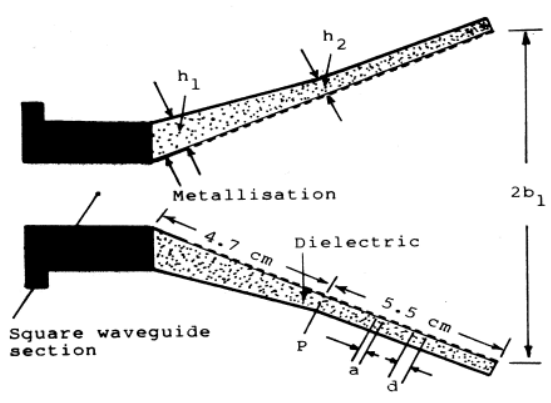

Horn HE

Fig.1. E-Plane view of the horn HI with interior throat tapering profile, horn HE with exterior throat tapering profile and their design details.

cost, heavy weight and tedious fabrication process. Alternate feed horns which simulate all the radiation characteristics of metallic corrugated horn antennas are reported [ 2,4,5 ]. However, in simulated scalar feed horns, [5] the tapered impedance matching profile was given to the interior of the E-plane walls. In the present paper, to explore further improvement in impedance matching and radiation characteristics, the tapered throat profile is given to the exterior side of the E-plane walls of the horn. For analysis, the radiation characteristics of the present horn (horn HE) are com compared with that of an identical horn (horn HI) with interior tapered throat profile. The E-plane view of horn HI with interior throat profile, horn HE with exterior throat tapering profile and their design details are shown in figure. 1.

\section{Design details of horn HE}

The horn HE is a square pyramidal horn with aperture dimensions $\mathrm{a}_{1}=\mathrm{b}_{1}=5.9 \mathrm{~cm}$. The $\mathrm{E}$ and $\mathrm{H}$-plane slant lengths are $\rho_{\mathrm{E}}=\rho_{\mathrm{H}}=12.8 \mathrm{~cm}$. The corresponding semi flare angles in the above planes are $\Psi_{\mathrm{E}}=\Psi_{\mathrm{H}}=25^{\circ}$. At the throat region, after $1 \mathrm{~cm}$ metalized portion, the dielectric thickness $\left(\mathrm{h}_{1}\right)$ is $\lambda / 2 \sqrt{\varepsilon_{\mathrm{r}}}-1$. This tapers to $\mathrm{h}_{2}=$ $\lambda / 4 \sqrt{\varepsilon_{\mathrm{r}}-1}$ at the point P. From the point $\mathrm{P}$ to the aperture of the horn, the thickness is kept constant at $\lambda / 4 \sqrt{\varepsilon_{\mathrm{r}}-1}$ so that balanced hybrid mode is satisfied at $8.4 \mathrm{GHz}$. The a/d value is kept at 0.5 .

\section{Experimental Results}

The variations of the return-loss of the two horns HI and HE with frequency are compared in figure 2. The comparative study shows that the abrupt angular variation caused by the interior tapered throat profile structure at the throat region of the horn $\mathrm{HI}$ is not degrading its return-loss characteristics as expected. In the 
entire $\mathrm{x}$-band of frequency, the maximum return-loss level exhibited by the horn $\mathrm{HI}$ is $-19.2 \mathrm{~dB}$, whereas by the horn $\mathrm{HE}$ is $-18.6 \mathrm{~dB}$.

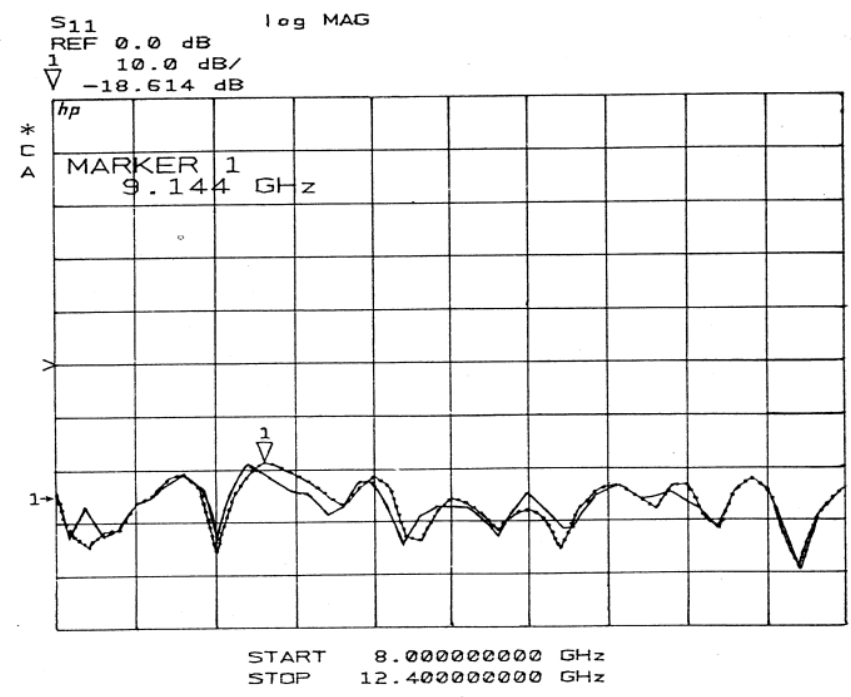

Fig.2. the variations of the return-loss of the two horns HI and HE with frequency. Horn HI Horn HE figure 3

The typical E-plane radiation patterns of the horns $\mathrm{HI}$ and $\mathrm{HE}$ at different frequencies are shown in
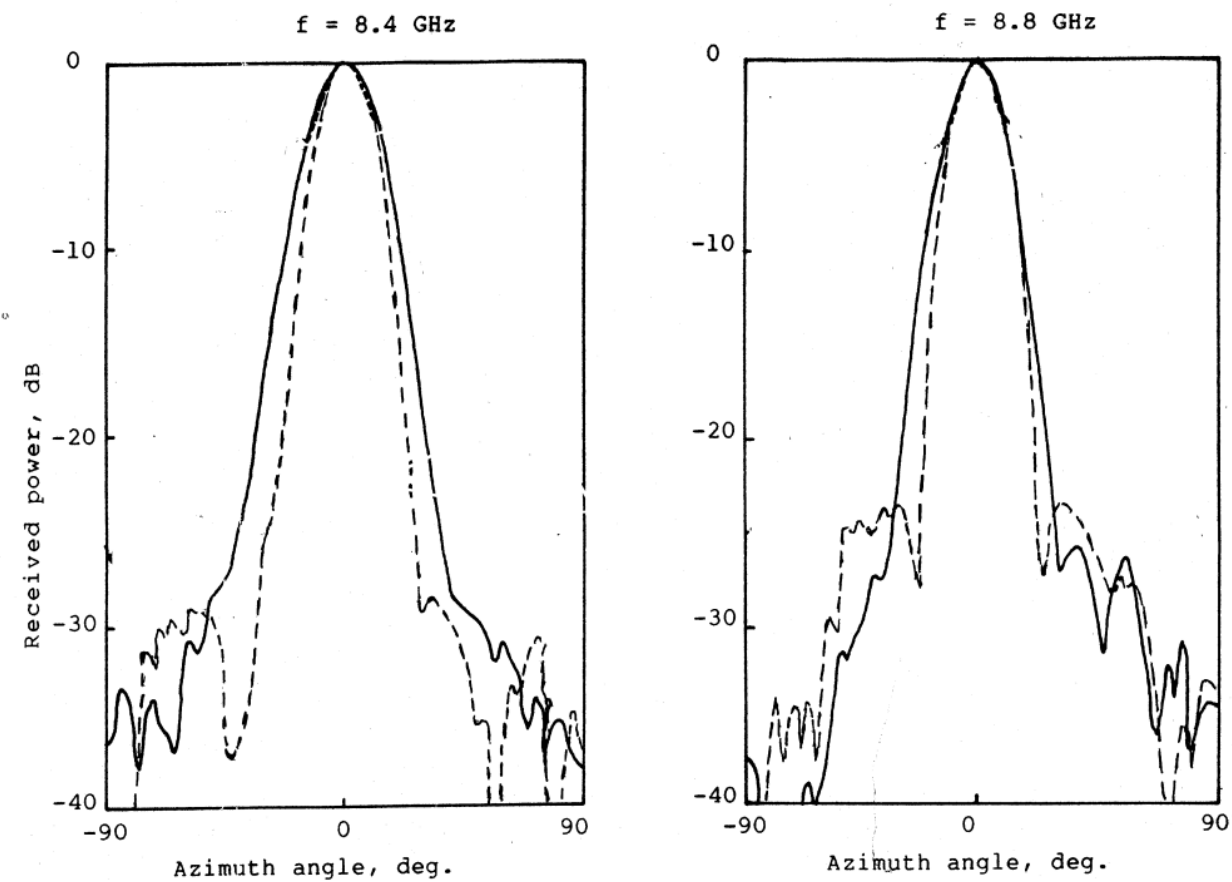

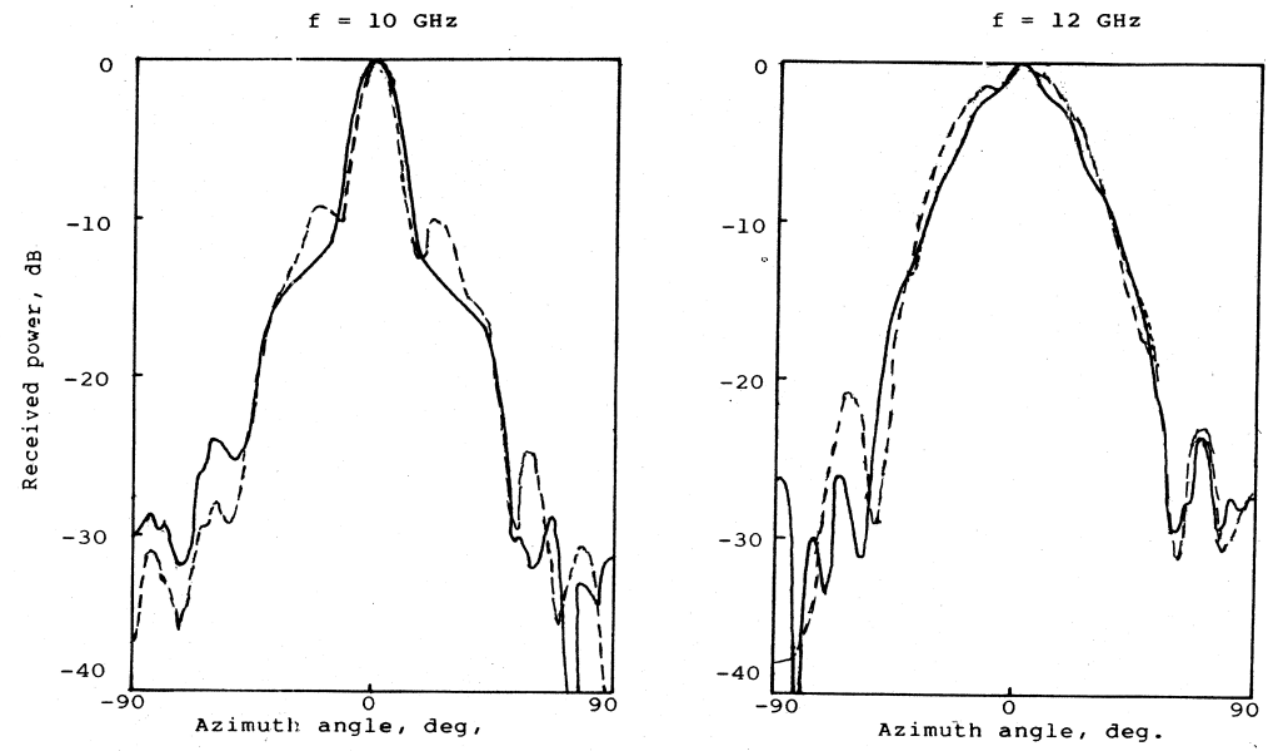

Fig .3. The typical E-plane radiation patterns of the horns $\mathrm{HI}$ and $\mathrm{HE}$ at different frequencies Horn HI Horn HE

The variation of side lobe levels and back lobe levels with frequency of both the horns is shown in figures 4 and 5. At the design frequency, both the horns $\mathrm{HI}$ and $\mathrm{HE}$ exhibits very low side lobe levels. As frequency increases, the side lobe levels of the two horns are found to be increasing. However, at higher frequencies, in the radiation pattern of horn HI, the presence of shoulder lobes are observed. The level ofthe shoulder lobes are found to be increasing with frequency. At $11 \mathrm{GHZ}$, the observed shoulder level of this horn is $-3.2 \mathrm{~dB}$ and it is increased to $-1.25 \mathrm{~dB}$ at $12 \mathrm{GHz}$. Though the side lobes of the horn $\mathrm{HE}$ are also increasing with frequency, such shoulder lobes are not observed in its radiation patterns. At higher frequencies, even though it shows "a bottle-neck nature" (BNN) in its radiation patterns, the $3 \mathrm{~dB}$ beam widths are found to be reduced. The BNN nature of the patterns may be due to the merging of shoulder lobes with the main lobe. The H-plane radiation patterns of both the horns are found to be identical to that of a conventional horn of same dimensions.

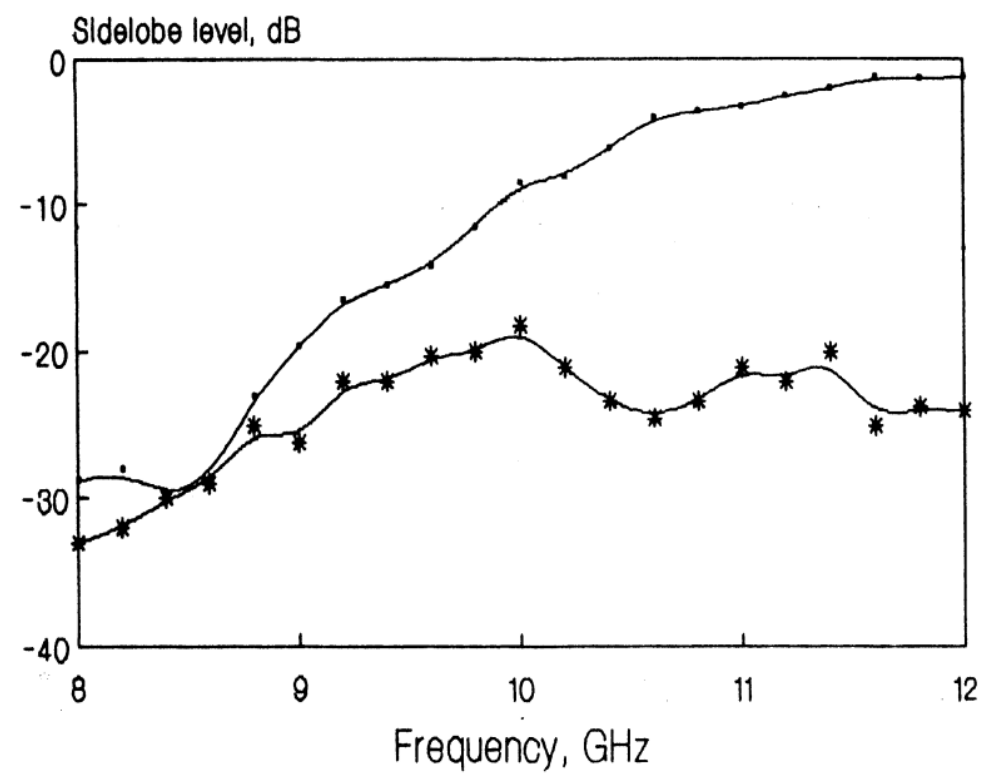

Fig.4. The variation of side lobe levels with frequency of both the horns $\mathrm{HI}$ and $\mathrm{HE}$

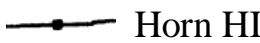

* - Horn HE 


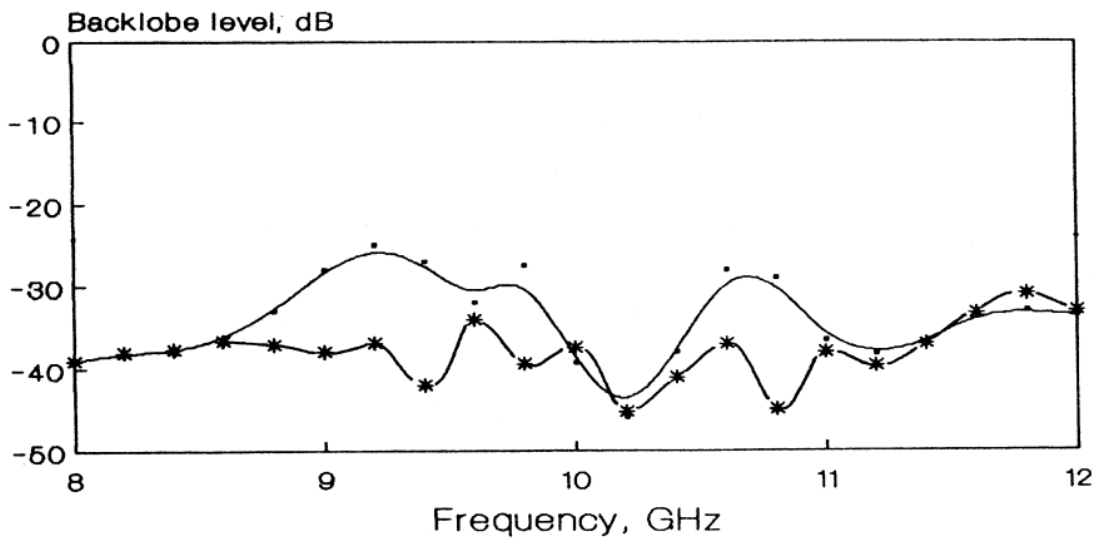

Fig.5. The variation of back lobe levels with frequency of both the horns $\longrightarrow$ Horn $\rightarrow$ *-Horn HE

For both the horns, throughout the frequency range, the expected beam symmetry is not observed. Compared to horn HI, horn HE shows a better back lobe characteristics. The frequency response of cross-polarization of both the horns is presented in figure 6. At the design frequency, horn HI shows better characteristics than the horn HE. Abovedesign frequency, both the horns show almost same $-25 \mathrm{~dB}$ cross-polarization characteristics.

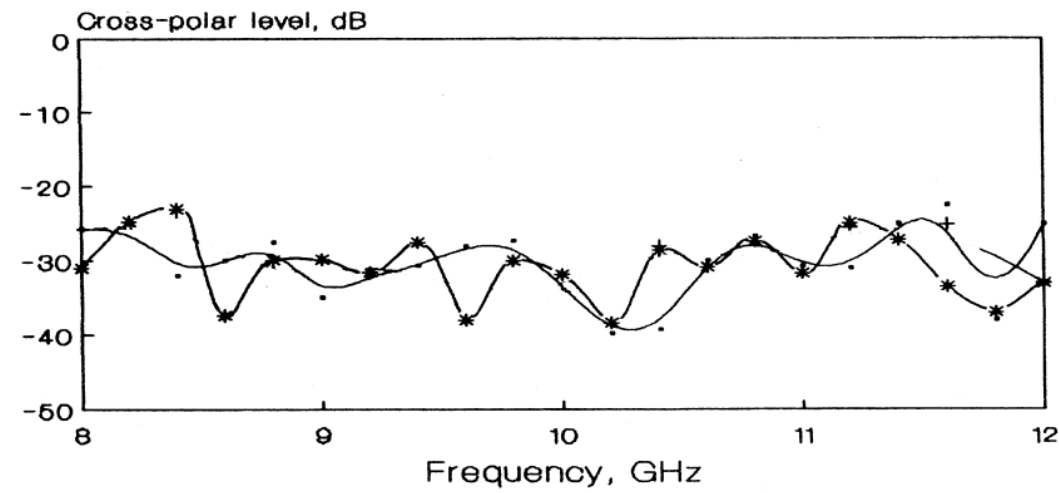

Fig.6. the variation of cross-polar levels with frequency of both the horns

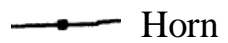

-*-Horn HE

The variation of 3-dB beam width of both the horns is shown in figure 7 . In the entire frequency range of interest, the 3-dB beam width of the horn $\mathrm{HE}$ is gradually decreasing with frequency. But in the case of horn $\mathrm{HI}$, from $8.4 \mathrm{GHz}$ to $10.8 \mathrm{GHz}$ its $3-\mathrm{dB}$ beam width is decreasing with frequency and above $10.8 \mathrm{GHZ}$, it is found to be increasing. The large beam widths at higher frequencies are attributed to the shoulder lobes of the radiation patterns.

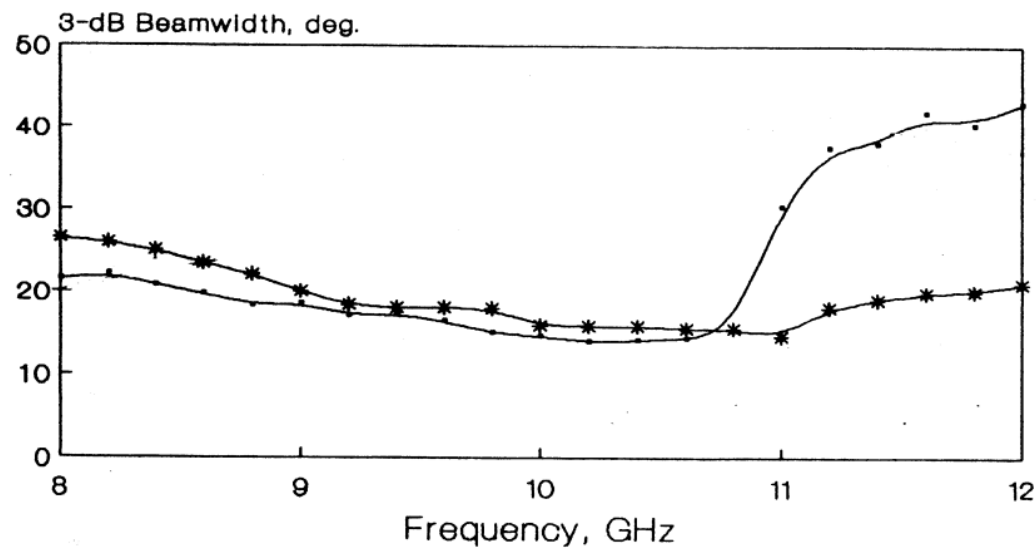

Fig.7. The variation of 3-dB beam width with frequency of both the horns $\mathrm{HI}$ and $\mathrm{HE}$ 
The variation of $10-\mathrm{dB}$ beam width with frequency of both the horns are presented in figure.8.In this casealso, up to $9.8 \mathrm{GHz}$, both the horns $\mathrm{HI}$ and $\mathrm{HE}$ show a decrease in beam width with frequency

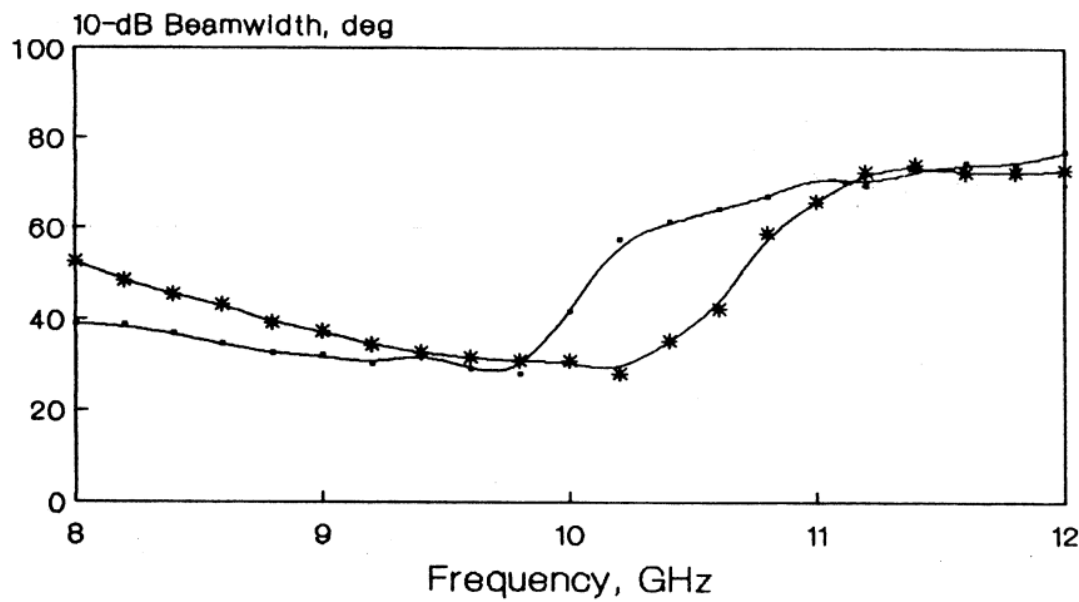

Fig. 8.The variation of 10-dB beam width with frequency of both the horns $\mathrm{HI}$ and $\mathrm{HE}$ $\longrightarrow$ Horn $\rightarrow$-Horn HE

and above this frequency, the beam widths are increasing considerably.

The frequency response of gain of the horns is presented in figure 9. As expected, in the lower part of the frequency band horn HI shows better gain characteristics than the horn HE whereas in the upper part the horn $\mathrm{HE}$ shows gain improvement.

\section{Conclusion.}

From the detailed comparative study of the radiation characteristics of the horn HI with interior tapered throat profile structure and the horn $\mathrm{HE}$ with exterior tapered throat profile structure, it is under stood that, the return loss characteristics of the horn $\mathrm{HI}$ is not seriously affected by the abrupt angular change at the throat region. However, compared to horn $\mathrm{HI}$, the horn $\mathrm{HE}$ shows considerable improvements in radiation characteristics such as side lobe and back lobe levels. The $-25 \mathrm{~dB}$ cross-polarization level of the horn HE is also almost identical to the horn HI. Due to the absence of shoulder lobes, compared to horn HI, the horn HE shows a gradual variation in its 3-dB and 10-dB beam widths with frequency. Except in the lower edge of frequency band of study, horn HE exhibits gain improvement than horn HI.

\section{References}

[1] A.Kumar, "Dielectric Lined WaveguideFfeed”, IEEE Trans. Antennas Propagat., Vol. AP-27, No.2 ,pp.279-282, March 1979.

[2] E.Lier and T.S Petterson,“ The Strip Loaded Hybrid Mode Feed Horn”, ibid., Vol. AP-36, No.(9), pp.1086-1088, Sept. 1987.

[3] E.Lier and P.S Kildal, "Soft and Hard Horn Antennas", ibid., Vol.36, No.8, pp.1152-1157, Aug. 1988.

[4] S tephen Rodrigues, P.Mohanan and K.G Nair“ Simulated Corrugated Feed horn Antenna”, Proc. IEEE AP-S Intl. Symp. as,USA,1990, pp. $984-987$

[5] S tephen Rodrigues, P.Mohanan and K.G Nair" A Strip Loaded Feed -Horn Antenna" IEEE Microwave and Guided wave Letters, Vol. 1, No.11, pp. 318-319, Nov. 1991

[6] P.J.B Clarricoats and A.D Olver, (Eds): "Corrugated Horns for Microwave Antennas", Peter Peregrinus Ltd., London, U.K, 1984.

[7] R.Chatterjee, "Dielectric and Dielectric Loaded Antennas" John Wiley and Sons Inc., Newyork, 1985. 